\title{
Fast Processing Approach for Near-Field Terahertz Imaging with Linear Sparse Periodic Array
}

\author{
Amir Masoud Molaei, Shaoqing Hu, Member, IEEE, Vasiliki Skouroliakou, Vincent Fusco, \\ Fellow, IEEE, Xiaodong Chen, Fellow, IEEE, and Okan Yurduseven, Senior Member, IEEE
}

\begin{abstract}
The benefits of terahertz (THz) radiation have increased its use, especially in imaging systems. Recently, the use of a linear sparse periodic array (SPA) has been proposed as an effective solution for two-dimensional (2D) scanning in THz imaging systems. However, the special multistatic structure of the SPA is such that it is not possible to apply fast Fourier transform-based techniques directly in the near-field (NF). Therefore, in this paper, a fast processing approach based on two Fourier techniques compatible with linear SPA is presented for NF THz imaging. In this approach, we first employ a multistatic-to-monostatic conversion to reduce phase errors due to NF multistatic imaging. Then, to improve the quality of the

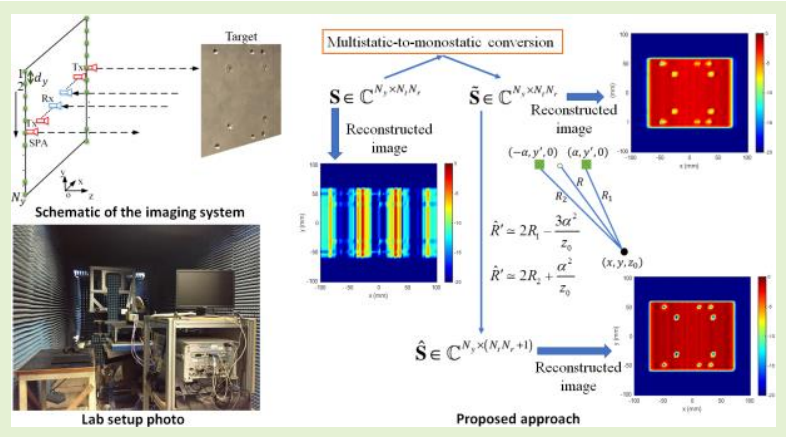
results, we mathematically derive an interpolation formula to counteract the non-uniform spacing of the virtual array. The modified data is then processed by three rapid techniques (fast Fourier transform (FFT)-inverse fast Fourier transform, matched filtering and a novel 1D FFT-based technique with low computational complexity) to obtain reconstructed images of the scene. Numerical and experimental results confirm the satisfactory performance of the proposed approach in terms of both computational time and the quality of the reconstructed image.
\end{abstract}

Index Terms-Fast processing, near-field, reduced dimension Fourier, sparse periodic array, THz imaging.

\section{INTRODUCTION}

$\mathrm{I}_{\mathrm{b}}^{\mathrm{N}}$ $\mathrm{N}$ recent years, terahertz $(\mathrm{THz})$ imaging technology has $I_{\text {been }}$ used in security screening, clinical and medical applications, pharmaceutical, food and agricultural industries, non-destructive testing and automotive to solve real-world challenges [1-3]. One of the most prominent advantages of $\mathrm{THz}$ radiation is its ability to penetrate a wide range of materials (fabric, plastic, wood, paper, semiconductors, etc.). Since $\mathrm{THz}$ photon energy is approximately six times smaller than an x-ray photon, its interaction with matter, especially with biological tissues, does not cause any detectable damage [4]. Also, compared to the microwave range, $\mathrm{THz}$ waves with shorter wavelengths provide much better imaging resolution.

For two-dimensional (2D) scanning, an active radar may be

This work was funded by the Leverhulme Trust under Research Leadership Award RL-2019-019.

Amir Masoud Molaei, Vasiliki Skouroliakou, Vincent Fusco and Okan Yurduseven are with the Institute of Electronics, Communications, and Information Technology (ECIT), Queen's University Belfast, Belfast BT3 9DT, U.K. (e-mails: a.molaei@qub.ac.uk; vskouroliakou01@qub.ac.uk; v.fusco@ecit.qub.ac.uk; okan.yurduseven@qub.ac.uk).

Shaoging $\mathrm{Hu}$ is with the College of Engineering, Design and Physical Sciences, Brunel University London, Uxbridge UB8 3PH, U.K. (e-mail: shaoqing.hu@brunel.ac.uk).

Xiaodong Chen is with the School of Electrical Engineering and Computer Science, Queen Mary University of London, London E1 4NS, U.K. (e-mail: xiaodong.chen@qmul.ac.uk). considered as a transmitter-receiver antenna pair (Tx-Rx) in monostatic mode [5]. In this case, by observing the Nyquist criterion in spatial sampling, images of the scene can be reconstructed with low or negligible distortion. However, such 2D mechanical scanning takes a lot of time to acquire data, which is a major drawback in real-time applications. A common and more time-effective approach is to perform 1D scanning with a linear array [6-8]. In a monostatic array, a large number of antenna elements are required to scan a relatively large scene (e.g., a human-scale scene) satisfying the Nyquist criterion (which is related to the inter-element spacing in terms of wavelength $\lambda$ ). This problem is more acute for the $\mathrm{THz}$ band and its implementation is sometimes impractical. For example, to scan a scene with a width of $0.3 \mathrm{~m}$ at $220 \mathrm{GHz}$ and an inter-element spacing of $\lambda / 2,441$ elements will be required. Multiple-input multiple-output (MIMO) apertures, also known as multistatic sparse arrays, offer an alternative solution to reduce the number of elements. Experiments have shown that a high-quality image can be obtained at $75-90 \mathrm{GHz}$ band by using a multistatic imaging system with a linear array consisting of 58 elements with an inter-element spacing of $\lambda$ [9]. However, implementing such a setup is still costly for experimental work at the $\mathrm{THz}$ band.

To overcome the above issues, $\mathrm{Hu}$ et al. have recently proposed a THz imaging system using a linear sparse periodic array (SPA) with large inter-element spacing [10-12]. To reconstruct the image, they use the generalized synthetic 
aperture focusing technique (GSAFT). This technique calculates the vectors between the position of each Tx (and $\mathrm{Rx}$ ) in each mechanical scanning step, and each target pixel, by discretizing the target space into several pixels. Although a high quality image can be obtained by defining a sufficient number of pixels, this technique has a high computational time.

In real-time imaging applications (e.g., concealed threat detection in high foot traffic environments), reducing the processing time is an important factor. However, the special multistatic structure of the SPA is such that it is not possible to apply fast Fourier transform (FT)-based techniques in the near-field (NF). Therefore, in this paper, a fast processing approach based on Fourier techniques compatible with linear SPA is presented for NF THz imaging. In this approach, we first employ a multistatic-to-monostatic conversion to reduce phase errors due to NF multistatic imaging. In contrast to conventional synthetic aperture radar (SAR) based solutions employing uniform sampling, using this conversion is not a trivial task for the sparse imaging problem. This is due to the fact that the effective aperture is non-uniformly sampled for our problem. To improve the quality of the results, we mathematically derive a phase interpolation formula intending to counteract the non-uniform spacing of the virtual array. In Section IV, we will show how the quality of the results in the case of SPA declines without applying this extracted phase approximation. It should be noted that conventional SAR reconstruction techniques cannot work with data that has not been processed by the above steps; and state-of-the-art algorithms such as [11] that can work with this data are computationally expensive. Following, we first show that conventional Fourier-based image reconstruction techniques such as fast Fourier transform-inverse fast Fourier transform (FFT-IFFT) and matched filtering are fully consistent with the proposed approach. We also present a new Fourier-based technique employing dimensionality reduction that further reduces the computational complexity in comparison to regular FFT-based solutions. We demonstrate qualitatively (by means of reconstructed images) and quantitatively (by means of computational complexity and error analyses) that the proposed approach can significantly reduce the complexity and increase the reconstruction speed while maintaining the visual quality of the reconstructed images. To evaluate the effectiveness of the proposed approach, we test the proposed techniques on both numerical and experimental data. Given that research in $\mathrm{THz}$ imaging is still very limited in terms of algorithm development, the proposed approach could provide new avenues for future research.

The main contributions and novelties of this paper are summarized below:

- Developing multistatic-to-monostatic conversion for SPA: As mentioned earlier, the use of SPA for $\mathrm{THz}$ frequencies is a major advantage due to offering large aperture sizes with fewer elements than is required for conventional arrays. In this paper, the feasibility of using multistatic-to-monostatic conversion for SPA is demonstrated.

- Mathematical derivation of a phase approximation to ensure the compatibility of Fourier-based solutions with the imaging scenario involving NF data collected by a sparse non-uniformly distributed MIMO effective aperture.

- Derivation of a new Fourier-based closed-form expression leveraging dimensionality reduction for image reconstruction with low computational complexity.

- Validation of the performance of the proposed approach in various experiments with both numerical and experimental data.

The rest of this paper is organized as follows: In Section II, the system model is described; Section III details the proposed approach; Section IV provides numerical results and experimental verifications; Finally, Section V presents the concluding remarks.

\section{SYSTEM MODEL}

Fig. 1 shows the general structure of the imaging system in the proposed approach. A linear SPA for electronic scanning is placed horizontally ( $x$-axis). The echo data of each Tx-Rx interaction can be captured by various channel access methods (including in its simplest form as time-division multiplexing (TDM)). The array then moves to the next vertical sampling step (y-axis). The sampling steps are uniformly distributed along the vertical axis to form the $2 \mathrm{D}$ aperture.

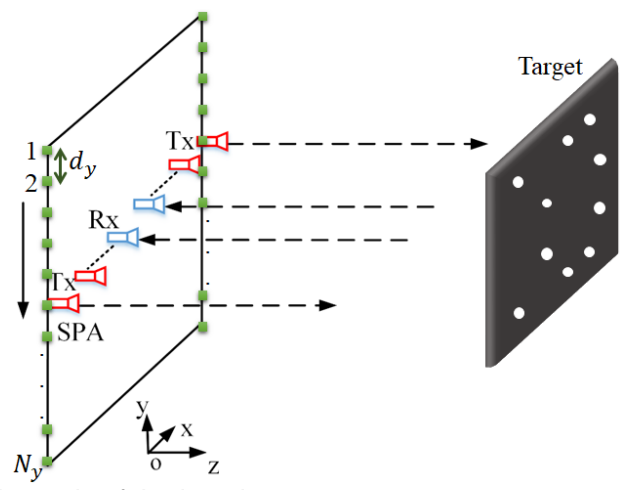

Fig. 1. Schematic of the imaging system.

A linear array configuration proposed in $[10,11]$ includes $N_{t}$ Tx elements with uniform spacing $d_{t}$ (distributed as two equal parts on both sides of the array) and $N_{r}$ Rx elements with uniform spacing $d_{r}=0.5 N_{t} d_{t}$ (in the middle). Fig. 2 shows two different setups of this configuration. In Setup 1, the Tx and Rx sensors are located across a line, while in Setup 2 there is a vertical distance between the Tx sensors and the $\mathrm{Rx}$ elements. We use both of these setups in this work.

\section{The Proposed Approach}

\section{A. Virtual Array Structure}

Under far-field (FF) assumptions, a multistatic array topology with $N_{t}+N_{r}$ physical antennas can be transformed into a virtual monostatic array with $N_{t} N_{r}$ elements using the effective phase center principle [13]. Thus, each of the configurations in Fig. 2 is equivalent to a denser linear virtual array of length $L_{t r}=\left(L_{t}+L_{r}\right) / 2$ consisting of $N_{t} N_{r}$ sampling points, where $L_{t}=N_{t} d_{t}+\left(N_{r}-1\right) d_{r}$ and $L_{r}=\left(N_{r}-1\right) d_{r}$ (see 
Fig. 3). $L_{t r}$ depicts the area that can be seen by the imaging system, also known as the field of view (FOV). It should be emphasized that regardless of whether $N_{r}$ is even or odd, there will be a gap of $d_{t}$ in the center of the virtual array. This is because the virtual element closest to the center of the array, whose position we represent with $(0, y, 0)$ for simplicity and to preserve symmetry, is created by the interaction between the closest $\mathrm{Tx}$ to the center (in position $x=L_{r} / 2+d_{t}$ (or $x=-L_{r} / 2-d_{t}$ ) and the farthest $\mathrm{Rx}$ from it (in position $x=-L_{r} / 2\left(\right.$ or $\left.\left.x=L_{r} / 2\right)\right)$ in Fig. 2 .

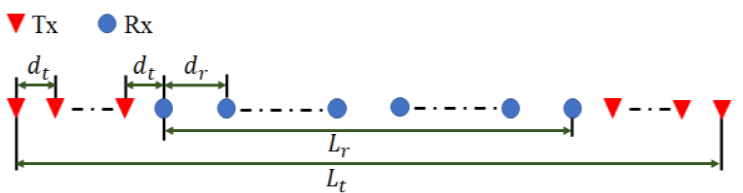

(a)

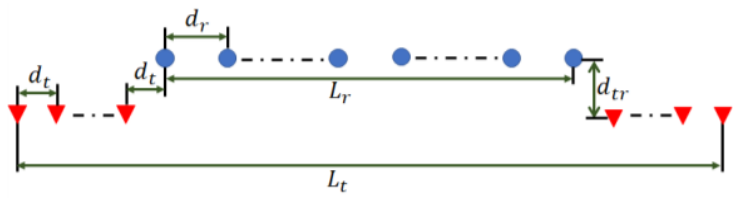

(b)

Fig. 2. Element distributions of SPA; (a) Setup 1, (b) Setup 2.

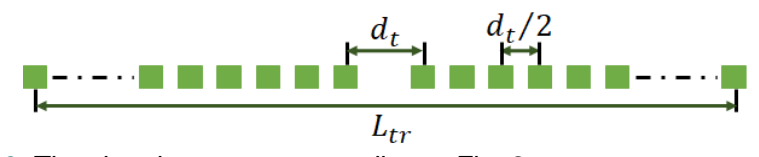

Fig. 3. The virtual array corresponding to Fig. 2.

\section{B. Multistatic-to-Monostatic Conversion}

Since we are dealing with a NF multistatic imaging system, we need a more accurate model to adapt FT-based fast image reconstruction techniques. This is because even if we ignore the gap in the center of the virtual array, in a NF multistatic scenario, the other uniform distances in Fig. 3 will not actually be realized because the virtual array model can be considered accurate only in the FF region.

Since Fourier-based image reconstruction methods require uniform spatial sampling, we first use a multistatic-tomonostatic conversion [14] for the captured raw data $\mathbf{S} \in \mathbb{C}^{N_{y} \times N_{t} N_{r}}$. By using this conversion, the received data phase of each Tx-Rx interaction is modified according to the position of the physical antennas and the corresponding virtual element (see [13-15] for more details). Suppose $\left(x_{C}, y_{C}, 0\right)$ is the position of the phase center corresponding to the transmitter element at $\left(x_{T}, y_{T}, 0\right)$ and the receiver element at $\left(x_{R}, y_{R}, 0\right)$. The multistatic data set can be converted to an effective monostatic version as follows:

$$
\tilde{s}\left(x_{C}, y_{C}\right)=s\left(x_{T}, y_{T}, x_{R}, y_{R}\right) \frac{s_{o}\left(x_{c}, y_{c}\right)}{s_{o}\left(x_{T}, y_{T}, x_{R}, y_{R}\right)},
$$

where $s_{o}\left(x_{C}, y_{C}\right)$ and $s_{o}\left(x_{T}, y_{T}, x_{R}, y_{R}\right)$ correspond to the monostatic and multistatic reference signals, respectively, and can be calculated as follows:

$$
\begin{aligned}
& s_{o}\left(x_{C}, y_{C}\right)=e^{-j 2 k_{0} R_{C}}, \\
& s_{o}\left(x_{T}, y_{T}, x_{R}, y_{R}\right)=e^{-j k_{0}\left(R_{T}+R_{R}\right)},
\end{aligned}
$$

where $R_{T}$ and $R_{R}$ are the distances between the Tx and $\mathrm{Rx}$ physical antennas to the reference point in the target domain, respectively, and $R_{C}$ is the distance between the corresponding virtual element and the reference point. $k_{0}=2 \pi f_{0} / c$ is the wavenumber corresponding to the carrier frequency $f_{0}$, and $c$ is the speed of light.

Note that we are still physically faced with a multistatic topology, however, the above conversion provides an artificial (mathematical) approximation of the corresponding monostatic topology. We refer to the converted data as $\tilde{\mathbf{S}} \in \mathbb{C}^{N_{y} \times N_{t} N_{r}}$.

\section{Phase Interpolation in the Virtual Array Center}

Due to the inconsistent gap in the center of the virtual array (see Fig. 3), the data $\tilde{\mathbf{S}}$ is not yet readily compatible with Fourier-based image reconstruction techniques. Our goal here is to interpolate the phase of the received signal in the center of the array based on the phase of the received signal at adjacent points. Let us denote the coordinates of the point scatterer and the two virtual elements closest to the center of the array with $\left(x, y, z_{0}\right),\left(\alpha, y^{\prime}, 0\right)$ and $\left(-\alpha, y^{\prime}, 0\right)$, respectively (see Fig. 4). Therefore, the corresponding distances can be calculated as follows:

$$
\begin{aligned}
& R=\sqrt{x^{2}+\left(y-y^{\prime}\right)^{2}+z_{0}^{2}}, \\
& R_{1}=\sqrt{(x-\alpha)^{2}+\left(y-y^{\prime}\right)^{2}+z_{0}^{2}}, \\
& R_{2}=\sqrt{(x+\alpha)^{2}+\left(y-y^{\prime}\right)^{2}+z_{0}^{2}} .
\end{aligned}
$$

Hence, $R$ can be rewritten in terms of $R_{1}$ as follows:

$$
R=\sqrt{R_{1}^{2}-\alpha^{2}+2 \alpha x}
$$

As a result, the total round-trip distance associated with the center of the array can be calculated as follows:

$$
R^{\prime}=2 R=2 \sqrt{R_{1}^{2}-\alpha^{2}+2 \alpha x} .
$$

By applying Taylor series expansion [16] with respect to $\alpha$ around zero, we have

$$
R^{\prime}(\alpha)=R^{\prime}(0)+\alpha \frac{\partial R^{\prime}}{\partial \alpha}(0)+\frac{1}{2 !} \alpha^{2} \frac{\partial^{2} R^{\prime}}{\partial \alpha^{2}}(0)+\ldots
$$

By performing the necessary calculations on (5), we have 


$$
\begin{aligned}
& R^{\prime}(0)=2 R_{1}, \\
& \frac{\partial R^{\prime}}{\partial \alpha}(0)=\frac{2 x}{R_{1}}, \\
& \frac{\partial^{2} R^{\prime}}{\partial \alpha^{2}}(0)=-\frac{2}{R_{1}}-\frac{2 x^{2}}{R_{1}^{3}} .
\end{aligned}
$$

By placing (7) into (6), we have:

$$
R^{\prime} \simeq 2 R_{1}-\frac{\alpha^{2}}{R_{1}}+\frac{2 \alpha x}{R_{1}}-\frac{\alpha^{2} x^{2}}{R_{1}^{3}} .
$$

By ignoring the last term (power of order 3 of the distance $R_{1}$ in the denominator parts), considering $(x-\alpha),\left(y-y^{\prime}\right) \ll z_{0}$, we can obtain an approximation of the total round-trip distance associated with the center of the array as follows:

$$
\hat{R}^{\prime} \simeq 2 R_{1}-\frac{3 \alpha^{2}}{z_{0}}
$$

where $\alpha=d_{t} / 2$. In a similar way, $\hat{R}^{\prime}$ can be approximated in terms of $R_{2}$ as

$$
\hat{R}^{\prime} \simeq 2 R_{2}+\frac{\alpha^{2}}{z_{0}} .
$$

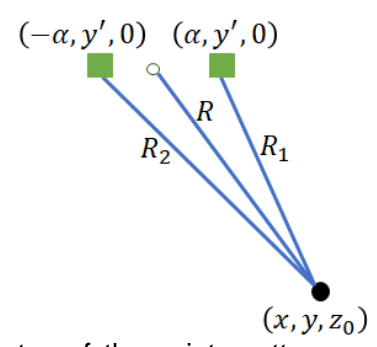

Fig. 4. The coordinates of the point scatterer and the two elements closest to the center of the array.

According to (5) and (9) (or (10)), the magnitude of the phase approximation error in the center of the virtual array can be obtained as follows:

$$
E=k_{0}\left|2 \sqrt{R_{1}^{2}-\alpha^{2}+2 \alpha x}-2 R_{1}+\frac{3 \alpha^{2}}{z_{0}}\right| .
$$

Fig. 5 shows the values of $E$ versus frequency for different ranges and $d_{t}$ values for the scanning midpoint and the reference point in the target domain. As can be seen, in all cases, with increasing frequency, the approximation error increases linearly, which is consistent with the findings of (11). Another point that can be deduced from Fig. 5 is that as the range increases, the error decreases (see solid lines). This means that the NF can be more challenging for phase approximations. However, the satisfactory results presented in Section IV show that the above approximation is effective even in the NF. It can also be found that increasing the inter- element spacing can lead to an increase in phase approximation error (see blue diagrams). This is because the approximate phase derived in the above equations is based on the phase of the side elements, so increasing (or decreasing) the distance of the elements directly leads to increasing (or decreasing) the approximation error.

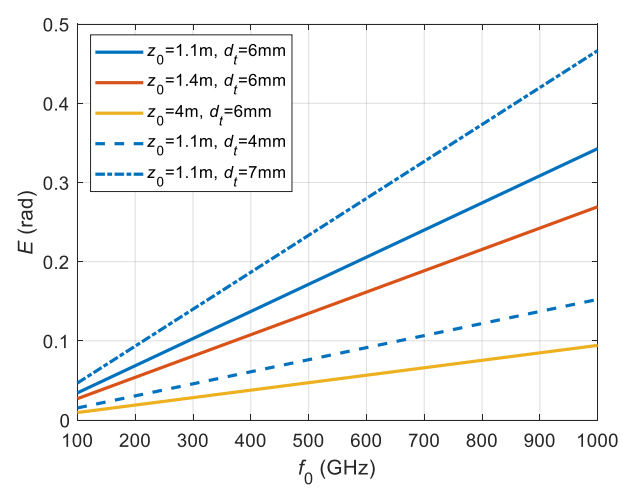

Fig. 5. The values of $E$ versus frequency for different ranges and $d_{t} s$.

By applying the phase interpolation to the data of the elements closest to the center of the array, the appropriate data for use in Fourier-based techniques is obtained, which we call $\hat{\mathbf{S}} \in \mathbb{C}^{N_{y} \times\left(N_{t} N_{r}+1\right)}$.

\section{Image Reconstruction}

Now, 2D target reflectivity can be reconstructed as [17]

$$
\begin{aligned}
& \rho(x, y)=F T_{2 D}^{-1}\left[F T_{2 D}\left[\hat{S}\left(k_{x}, k_{y}\right)\right] e^{j \sqrt{4 k_{0}^{2}-k_{x}^{2}-k_{y}^{2}} z_{0}}\right] \\
& k_{x}^{2}+k_{y}^{2} \leq 4 k_{0}^{2} .
\end{aligned}
$$

In a more straightforward way, the 2D target reflectivity can also be obtained using the matched filtering technique [18] as follows:

$$
\rho(x, y)=F T_{2 D}^{-1}\left[F T_{2 D}[\hat{s}(x, y)] F T_{2 D}\left[h_{z_{f}}^{*}(x, y)\right]\right],
$$

where $h_{z_{f}}(x, y)$ is the impulse response evaluated at the focusing distance $z_{f}$ instead of $z_{0}$. For a point target located at the origin of the target coordinate system $\left(0,0, z_{0}\right)$, the received reflected wave data is given by $[18,19]$

$$
h(x, y)=e^{-j 2 k_{0} \sqrt{x^{2}+y^{2}+z_{0}^{2}}} .
$$

where $h(x, y)$ is the impulse response of the imaging system. Although the latter technique does not consider any visibility region condition, analyses [18] show that the resolution depends on the correlation between $h(x, y)$ and $h_{z_{f}}^{*}(x, y)$. As the aperture size decreases, the image resolution decreases, because the correlation is no longer an impulse function [18].

In addition to the two techniques mentioned above, various versions of the range migration algorithm (RMA) are 
presented in the literature under various hypotheses and scenarios [14, 20-23]. Common steps in all RMA versions include multidimensional FTs, filtering, and Stolt interpolation. Aiming to reduce computational complexity, a new closed-form expression for image reconstruction is derived here, which uses 1D FFT computations with a much lower computational load than 2D FFT computations. It also does not require the heavy computational Stolt interpolation step. According to (1) and using the first Born approximation [24], the backscattered data associated with a distributed target can be written as follows:

$$
\tilde{s}\left(x_{T}, x_{R}, y_{C}\right) \simeq \int_{x} \int_{y} \rho(x, y) e^{-j 2 k_{0} \sqrt{\left(x-x_{C}\right)^{2}+\left(y-y_{C}\right)^{2}+z_{0}^{2}}} d y d x
$$

By using backpropagation imaging equation [25], the target image can be retrieved as follows:

$$
\begin{aligned}
& \rho(x, y)= \\
& \quad \int_{x_{T}} \int_{x_{R}} \int_{y_{C}} \tilde{s}\left(x_{T}, x_{R}, y_{C}\right) e^{j 2 k_{0} \sqrt{\left(x-x_{C}\right)^{2}+\left(y-y_{C}\right)^{2}+z_{0}^{2}}} d y_{C} d x_{R} d x_{T} .
\end{aligned}
$$

In the above equation, the inner integral can be considered as a convolution operation with respect to $y$ in the following form:

$$
\rho(x, y)=\int_{x_{T}} \int_{x_{R}} \tilde{s}\left(x_{T}, x_{R}, y\right) * e^{j 2 k_{0} \sqrt{\left(x-x_{C}\right)^{2}+y^{2}+z_{0}^{2}}} d x_{R} d x_{T} .
$$

By taking Fourier transform with respect to $y$ of both sides of the above equation, we have:

$$
\mathrm{P}\left(x, k_{y}\right)=\int_{x_{T}} \int_{x_{R}} \tilde{S}\left(x_{T}, x_{R}, k_{y}\right) H\left(x, x_{C}, k_{y}\right) d x_{R} d x_{T}
$$

where $H\left(x, x_{C}, k_{y}\right)$ is the Fourier transform of $h\left(x, x_{C}, y\right)=e^{j 2 k_{0} \sqrt{\left(x-x_{C}\right)^{2}+y^{2}+z_{0}^{2}}}$ with respect to $y$. The above equation states that for each $x$, the value of $\mathrm{P}$ must be calculated by considering all transmitters and receivers and their superposition; As a result, we can obtain the target image using the following closed-form expression:

$$
\begin{array}{r}
\rho(x, y)= \\
F T_{1 D, y}^{-1}\left[\sum_{i=1}^{N_{t}} \int_{x_{R}} F T_{1 D, y}\left[\tilde{s}_{x_{T_{i}}}\left(x_{R}, y\right)\right] F T_{1 D, y}\left[h_{x_{T_{i}}}\left(x_{i^{\prime}}, x_{C}, y\right)\right] d x_{R}\right], \\
i^{\prime}=1,2, \ldots, M_{x},
\end{array}
$$

where $\tilde{s}_{x_{x_{i}}}$ and $h_{x_{x_{i}}}$ denote the values of $\tilde{s}$ and $h$ corresponding to the $i$-th transmitter antenna, respectively. $M_{x}$ is the number of points used to discretize $x$. Obviously, the larger the $M_{x}$ value selected, the better the image resolution. This comes at the cost of increasing computations. Note that in the above equations, the transmitter and receiver notations can be easily swapped, meaning that in contrast to (19), outer and inner summations can be applied to the receiver and transmitter elements, respectively. Because the latter equation relies on 1D FFT calculations, we call it the reduced dimension Fourier (RDF) technique.

Here, we provide an analysis of the computational complexity of the FFF-IFFT, matched filtering, and RDF techniques, all three of which are based on the Fourier transform. The FFT technique requires a 2D FFT operation and a 2D FFT operation in addition to the Stolt interpolation operation [26]. Therefore, the major computational complexity in the FFT-IFFT technique is $\mathcal{O}\left(2 N_{F}^{2} \log N_{F}^{2}+2\left(2 N_{K}-1\right) N_{F}^{2}\right)$, where $N_{F}$ and $N_{K}$ denote the number of FFT points and the Stolt interpolation kernel length [27], respectively. The matched filtering technique mainly requires two 2D FFT operations and one 1D IFFT operation, and its computational complexity is $\mathcal{O}\left(3 N_{F}^{2} \log N_{F}^{2}+N_{F}^{2}\right)$. Unlike the other two techniques, the RDF technique does not involve any 2D FFT operations. It requires several 1D FFT operations commensurate with the number of transmitters (or receivers) and some multiplication operations. The major computational complexity of the RDF technique is $\mathcal{O}\left(\left(2 N_{t}+1\right) N_{F} \log N_{F}+N_{t} N_{r} N_{F} M_{x}\right)$. Fig. 6 shows the computational complexity of the various techniques as a function of $N_{F}$. As can be seen, the proposed RDF technique is generally less computationally complex than the other techniques. The main reason for this is the elimination of the 2D Fourier computations that have far more calculations than the 1D Fourier steps. It can also be seen that as the $N_{F}$ increases, the computational improvement of the RDF technique becomes significantly more pronounced. Note that as the number of FFT points increases, we expect the quality of the reconstructed images to improve.

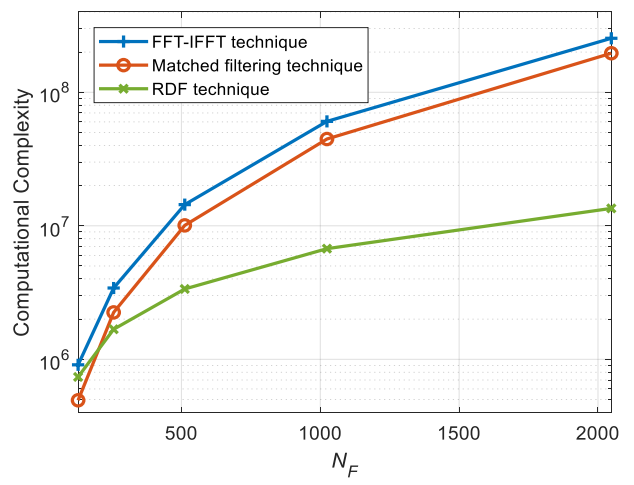

Fig. 6. Computational complexity of different techniques versus $N_{F}$. $M_{x}=101, N_{t}=8$ and $N_{r}=8$.

\section{Numerical and Experimental Results}

To evaluate the performance of the proposed approach, in this section, numerical and experimental results are presented. Numerical data are obtained from electromagnetic simulations in FEKO (see $[11,12]$ for more details). The values of the imaging system parameters are given in Table I. According to the values of the parameters, targets with distances less than 
approximately $54 \mathrm{~m}$ from the array are located in the NF [28]. Where not mentioned, $M_{x}$ is equal to 101. Colorbar in all figures is in $\mathrm{dB}$ scale. All computations are performed on the MATLAB R2020b of 64-bit Windows 10 operating system with $12 \mathrm{~GB}$ of random-access memory and a Core-i7 central processing unit at $2.7 \mathrm{GHz}$. In addition to the computational complexity analysis presented in Section III, this section will provide qualitative comparisons as well as quantitative analyzes such as computational time, resolution, and normalized mean squared error (NMSE) to examine performance. Computation formula for NMSE is as follows [29]:

$$
\mathrm{NMSE}=\frac{\sum_{i=1}^{M} \sum_{i^{\prime}=1}^{N}\left|\rho_{\mathrm{Rec}}\left(x_{i}, y_{i^{\prime}}\right)-\rho_{\mathrm{Ref}}\left(x_{i}, y_{i^{\prime}}\right)\right|^{2}}{\sum_{i=1}^{M} \sum_{i^{\prime}=1}^{N}\left|\rho_{\mathrm{Ref}}\left(x_{i}, y_{i^{\prime}}\right)\right|^{2}}
$$

where $\rho_{\text {Rec }}$ and $\rho_{\text {Ref }}$ denote the reconstructed image and reference image with $M \times N$ sizes, respectively.

TABLE I

IMAGING SYSTEM PARAMETERS FOR THE TARGET IN FIG. 7

\begin{tabular}{|c|c|c|c|c|c|c|c|c|}
\hline Parameter & $f_{0}$ & $N_{t}=N_{r}$ & $N_{y}$ & $d_{t}$ & $d_{r}$ & $d_{t r}$ & $d_{y}$ & $z_{0}$ \\
\hline Value & $220 \mathrm{GHz}$ & 8 & 76 & $\begin{array}{c}6 \mathrm{~mm} \\
(4.4 \lambda)\end{array}$ & $\begin{array}{c}24 \mathrm{~mm} \\
(17.6 \lambda)\end{array}$ & $181 \mathrm{~mm}$ & $4 \mathrm{~mm}$ & $1.1 \mathrm{~m}$ \\
& & & & & & \\
\hline
\end{tabular}

Fig. 7 shows a picture of a real target. It is a rectangular metal plate with a size of $145 \times 120 \times 5 \mathrm{~mm}^{3}$. The metal plate has several holes (diameters of six cylinder holes are $6 \mathrm{~mm}$ and $7 \mathrm{~mm}$; top and bottom diameters of four screw holes are $5 \mathrm{~mm}$ and $8 \mathrm{~mm}$ ). The SPA consists of the typical pyramidal horn, which has a half power beamwidth (HPBW) of about 42 degrees. As we will see, a large HPBW provides an ideal cross-range illumination of the scene, thus, we can focus more on the algorithm rather than the practical limitations caused by a narrow HPBW in terms of illuminating the FOV. Fig. 8 shows images of the raw echo data obtained from the two setups, Setup 1 and Setup 2 as depicted in Fig. 2 earlier. Fig. 9 shows the reconstructed images resulting from applying the FFT-IFFT operation (similar to (12)) directly to the raw data $\mathbf{S}$. As can be seen, the reconstructed images do not provide any meaningful visualization of the target. It can be clearly seen that the original data captured by the SPA cannot be processed by Fourier-based techniques due to non-compliance with the Nyquist criterion. As a next step, when we apply the FFT-IFFT operation to the converted data $\tilde{\mathbf{S}}$, the results give a good idea of the target (see Fig. 10). However, as can be seen, the results suffer from some distortion in the images; so that although some holes are detectable, there is not sufficient resolution for proper recognition. The reason for this, as mentioned in Section III, is the gap in the center of the virtual array. Finally, let us consider the images reconstructed by the proposed approach (after applying the conversion and interpolation to the raw data). As can be seen in Figs. 11(a) and 11(b), the results now provide not only a good idea of the target but also the details of the image are well recognizable. Note that the diameters of the holes in Fig. 7 are different; these differences can also be identified in the images in Figs. 11(a) and 11(b). An approximation of the theoretical crossrange resolution, when the target distance is much larger than scanning aperture length, can be calculated as $0.5 \lambda z_{0} / L_{t r}$ [19]. Therefore, according to the data in Table I, $L_{t r}$ is equal to $192 \mathrm{~mm}$ and as a result, the cross-range resolution is approximately equal to $3.9 \mathrm{~mm}$. So, it makes sense that the holes in Figs. 11(a) and 11(b) are well recognizable as they fall within the resolution limit of the synthesized aperture.

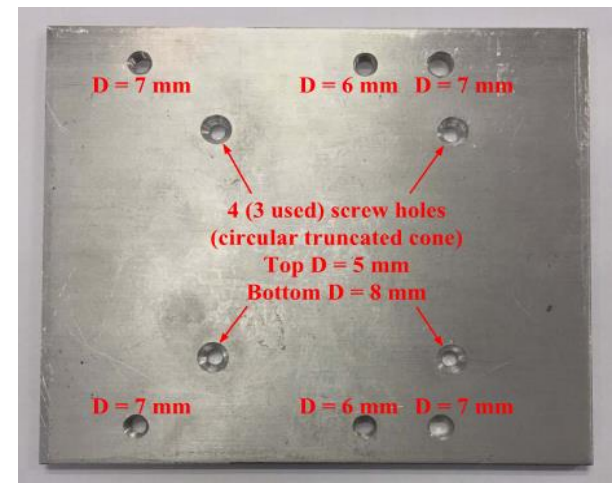

Fig. 7. First target photo [11]. All the results presented in Figs. 8-25 are related to this target profile.

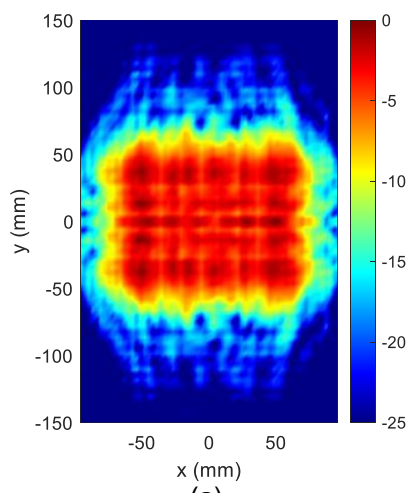

(a)

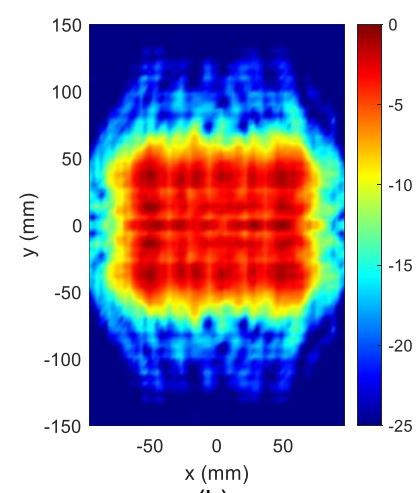

(b)
Fig. 8. Image of raw echo data; (a) obtained from Setups 1, (b) obtained from Setup 2.

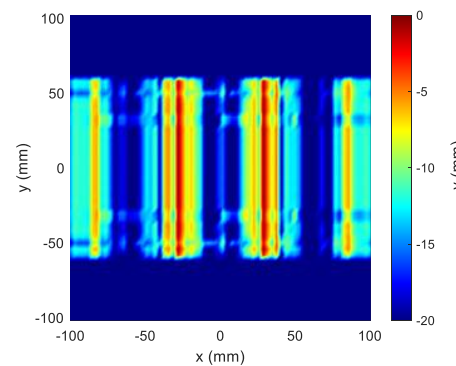

(a)

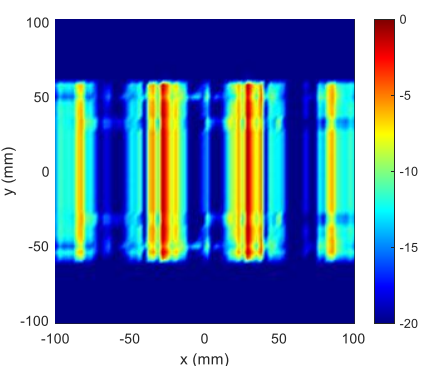

(b)
Fig. 9. Reconstructed images from applying FFT-IFFT operations directly to $\mathbf{S}$; (a) Setup 1, (b) Setup 2.

A careful analysis of Figs. 11(a) and 11(b) reveals that Fig. 11(a) is of somewhat better quality. This makes sense because, in Setup 2, we expect more phase errors. In fact, in Setup 1, the positional deviation of the virtual array elements relative to the physical antennas is only in the horizontal direction, while in Setup 2, there is a vertical deviation as well. Note that here the phase error due to vertical deviation is constant for all 
This article has been accepted for publication in a future issue of this journal, but has not been fully edited. Content may change prior to final publication. Citation information: DOI10.1109/TFUZZ.2022.3144448, IEEE Sensors Journal

elements (since the configuration of Setup 2 in the vertical direction can be considered as a quasi-monostatic state), while not in the horizontal direction. However, as can be seen, the results presented for both setups are in good agreement, indicating the ability to generalize and extend the proposed approach to 2D arrays. Figs. 11(c) and 11(d) show the images reconstructed by the proposed approach with the matched filtering technique. Compared to Figs. 11(a) and 11(b), i.e., images reconstructed by the proposed approach with the FFTIFFT technique, the image quality (especially for Setup 2) is slightly lower; however, we can achieve better computational performance by utilizing this technique. It should be noted that although the latter technique is more straightforward and faster to implement, the analyzes in [18] show that aperture size is a major limitation in the resolution of images reconstructed by this technique. Figs. 11(e) and 11(f) show the results related to employing the RDF technique in the proposed approach. As can be seen, the details of the scene are still well recognizable. Although the relative quality is lower than the corresponding images in Figs. 11(a) and 11(b), as shown in Fig. 6 and will be discussed further Figs. 11(e) and 11(f) are reconstructed more rapidly due to the reduced computational complexity of the RDF technique.

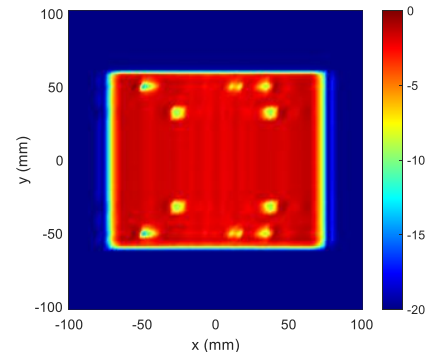

(a)

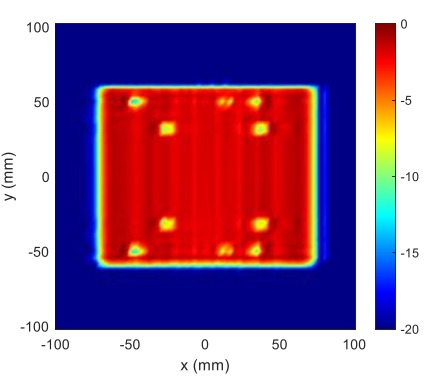

(b)
Fig. 10. Reconstructed images from applying FFT-IFFT operations to $\tilde{\mathbf{S}}$; (a) Setup 1, (b) Setup 2.

Since our experimental data is based on the standard horn, let us investigate here the effect of changing the beam properties on the results. To have a fair comparison with experimental results presented at the end of this section, here we have considered a WR3 band standard horn having about 12 degrees HPBW at $220 \mathrm{GHz}$ as the SPA element. Reconstructed images from the simulated numerical data can be seen in Figs. 11(g), 11(h) and 11(i). As can be seen, although the beamwidth has become narrower, the images have still been reconstructed correctly by all three Fourierbased techniques, but with a slight loss of quality. A more accurate equation for predicting cross-range resolution, which also takes into account the beamwidth effect, is given in [5, 11]. This equation shows that cross-range resolution is inversely proportional to HPBW. So, it makes sense to expect a better resolution for wide beam horn outputs than for standard horn outputs.

In the scenario of Figs. 11(a), 11(c) and 11(e), if we reduce $d_{t}$ to $4 \mathrm{~mm}$ (FOV reaches $128 \mathrm{~mm}$ ), the theoretical cross-range resolution deteriorates compared to the previous state (reaches $5.9 \mathrm{~mm}$ ), which is confirmed by the results in Fig. 12. The blurred edges of the target in Fig. 12 are created because, in the latter state, the FOV is slightly smaller than the width of the target.

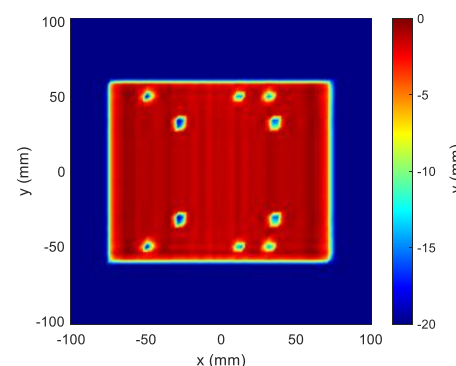

(a)

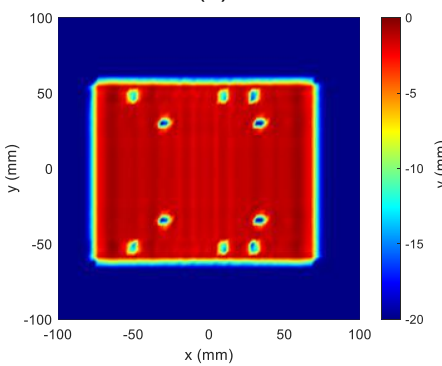

(c)

$(\mathrm{e})$

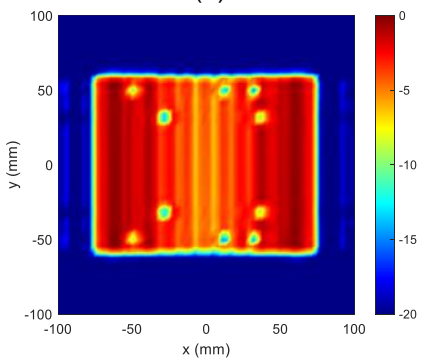

(g)

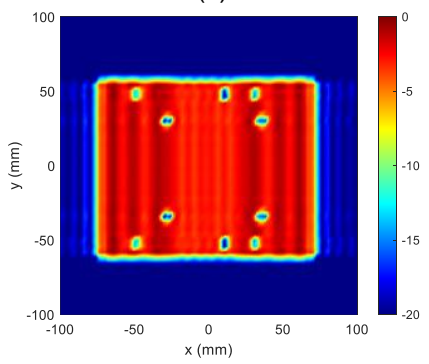

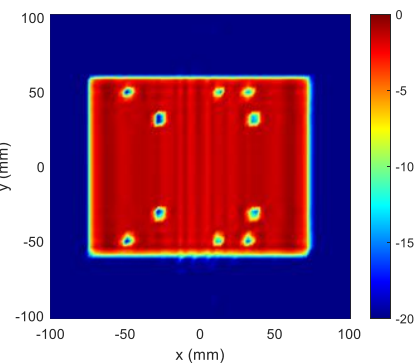

(b)

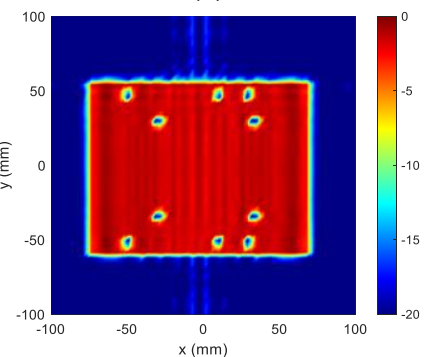

(d)

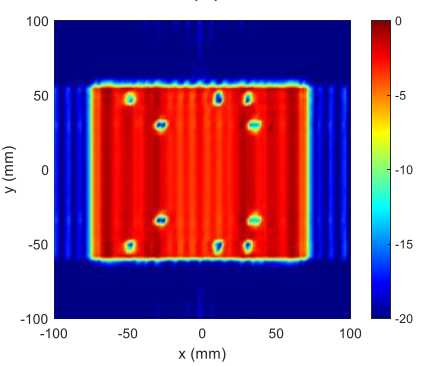

(f)

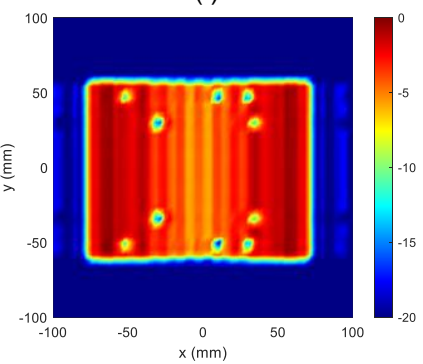

(h)

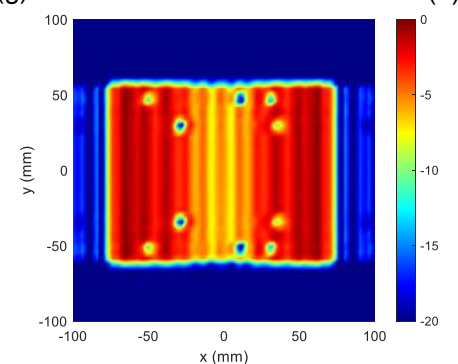

(i)

Fig. 11. Images reconstructed by the proposed approach; (a) with FFTIFFT technique, Setup 1, wide beam horn, (b) with FFT-IFFT technique, Setup 2, wide beam horn, (c) with matched filtering technique, Setup 1, wide beam horn, (d) with matched filtering technique, Setup 2, wide beam horn, (e) with RDF technique, Setup 1, wide beam horn, (f) with RDF technique, Setup 2, wide beam horn, (g) with FFT-IFFT technique, Setup 2, standard horn, (h) with matched filtering technique, Setup 2, standard horn, (i) with RDF technique, Setup 2, standard horn. 


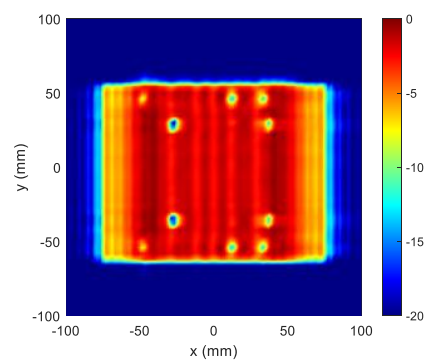

(a)

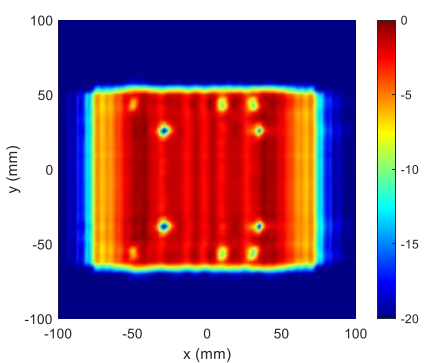

(b)

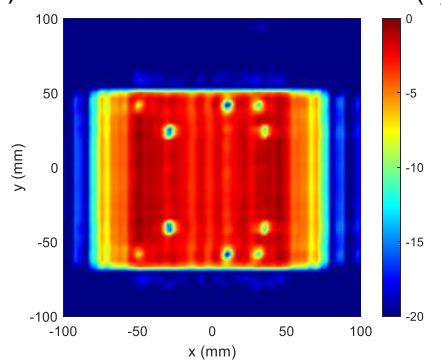

(c)

Fig. 12. Images reconstructed by the proposed approach using Setup 1 and wide beam horn when $d t$ is reduced; (a) with FFT-IFFT technique, (b) with matched filtering technique, (c) with RDF technique.

Fig. 13 shows the results obtained by the GSAFT technique presented in [11]. By comparing Figs. 11(a), 11(b), 13(a) and 13(b), it can be seen that the results processed by the proposed approach do not have the artifacts created in the images reconstructed using the GSAFT technique [11]. However, the shapes of the holes reconstructed using the approach presented in [11] seem to be slightly better defined. The reason for this is that, as mentioned in Section I, in the GSAFT approach [11], the distances from the target pixels to the elements are accurately calculated. In comparison, in our proposed approach, to provide a uniform spatial sampling artificially (mathematically), we approximate distances based on data modified by using multistatic-to-monostatic conversion and interpolation operations.

Although the quality of the reconstructed image has also been considered in this study, the main focus has been on accelerating computations for use in real-time applications. GSAFT is associated with a high computational time due to the calculation of numerous vectors between the position of the antennas and the discretized scene. This will be more acute for 3D imaging because the scene will have to be discretized with a huge number of voxels. Table II provides a comparison between the reconstruction time needed for Figs. 11 and 13. According to this comparison, in the case of Fig. 13, the reconstruction time is equal to 2.68 seconds (by defining the scene as a $101 \times 101$ pixel image); while the reconstruction time in the proposed approach is 0.22 and 0.14 seconds, respectively, with FFT-IFFT and matched filtering techniques. In fact, in this case, the proposed approach, in terms of computational time, has provided an almost 12- and 19-fold improvement, respectively, compared to [11]. By using the developed RDF technique, the reconstruction time is further reduced to 0.12 seconds, corresponding to an increase in the reconstruction speed by a factor of about 22 times in comparison to [11].

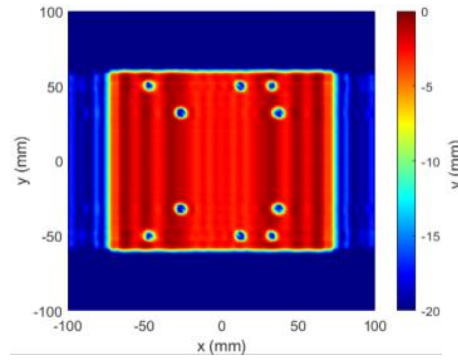

(a)

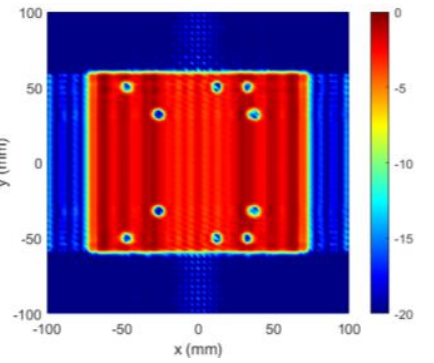

(b)

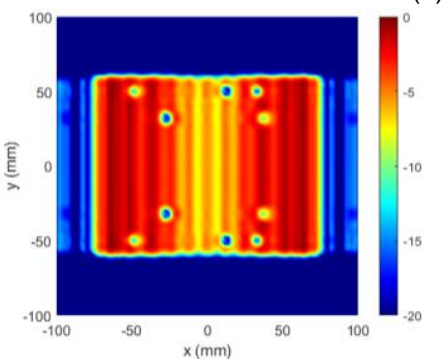

(c)

Fig. 13. Images reconstructed by the approach [11]; (a) using wide beam horn, Setup 1, (b) using wide beam horn, Setup 2, (c) using standard horn, Setup 2. The number of scene pixels $\left(N_{x} \times N_{y}\right)$ : $101 \times 101$.

TABLE II

COMPARISON OF COMPUTATIONAL TIMES FOR IMAGE RECONSTRUCTION BY DIFFERENT TECHNIQUES IN FIGS. 11 AND 13

\begin{tabular}{|c|c|c|c|c|}
\hline Figures & $\begin{array}{c}11(\mathrm{a}), 11(\mathrm{~b}) \\
\text { and } 11(\mathrm{~g})\end{array}$ & $\begin{array}{c}11(\mathrm{c}), 11(\mathrm{~d}) \\
\text { and } 11(\mathrm{~h})\end{array}$ & $\begin{array}{c}11(\mathrm{e}), 11(\mathrm{f}) \\
\text { and } 11(\mathrm{i})\end{array}$ & 13 \\
\hline $\begin{array}{c}\text { Computational } \\
\text { Time }\end{array}$ & $0.22 \mathrm{Sec}$ & $0.14 \mathrm{Sec}$ & $0.12 \mathrm{Sec}$ & $2.68 \mathrm{Sec}$ \\
\hline
\end{tabular}

The control parameters in the computational complexity of the GSAFT method and FFT processing are the number of pixels intended for scene discretization and $N_{F}$, respectively. In the case of the RDF technique, $M_{x}$ is another control parameter in addition to $N_{F}$. For a more comprehensive time study, in Fig. 14, we have calculated the computational time versus these control parameters. Obviously, with increasing the value of these parameters, the processing time has increased. Also, the findings in Fig. 14 are consistent with what can be deduced from Fig. 6. Fig. 15 shows a comparison between the quality of the reconstructed images in the proposed approach with the FFT-IFFT technique for $N_{F}=128$ and $N_{F}=1024$. A closer look at them reveals the slightly better image quality of Fig. 15(b), which is obtained by using more FFT points. Based on our investigations in this particular case, we found $N_{F}=2^{10}$ to be the upper-bound limit, beyond which the performance of the image reconstruction algorithm becomes saturated; in other words, after this value, increasing $N_{F}$ no longer has any significant effect on improving image quality. The results presented in Fig. 16 confirm this observation. In Fig. 16, the NMSE values calculated in the proposed approach with the FFT-IFFT technique can be seen for three different modes. The reference image is the image reconstructed by FFT-IFFT technique in first mode (Setup 1, wide beam horn) when $N_{F}=2048$. As expected, and the images in Fig. 11 confirm, the diagrams for 
setup 2 demonstrate more error; the standard horn diagram also indicates more error (meaning lower image quality) than the wide beam horn diagram. Fig. 17 shows a comparison between the quality of the reconstructed images in the proposed approach with the matched filtering technique for $N_{F}=108$ and $N_{F}=512$. We can still see the relative improvement in image quality by increasing the FFT points in Fig. 17. In Fig. 18, the NMSE values calculated in the proposed approach with the matched filtering technique can be seen for three different modes. The reference image is the image reconstructed by matched filtering technique in first mode (Setup 1, wide beam horn) when $N_{F}=512$. It is observed that in the case under study, after $N_{F}=2^{7}$, the results are saturated. A comparison of Figs. 11(c) and 17(b) confirms this. The corresponding results when using the RDF technique in the proposed approach are shown in Figs. 19 and 20. In the case of RDF, there are two control parameters $N_{F}$ and $M_{x}$, so NMSE diagrams are obtained versus both. The reference image for the diagrams in Fig. 20(a) is the image reconstructed by RDF technique in first mode (Setup 1, wide beam horn) when $N_{F}=512$ and $M_{x}=101$. Also, the reference image for the diagrams in Fig. 20(b) is the image reconstructed by RDF technique in first mode when $N_{F}=512$. Note that in the latter case, since the size of the data used in the reconstructed image varies by the value of $M_{x}$, the diagrams in Fig. 20(b) for each $M_{x}$ are obtained based on a comparison with the reference image in the same $M_{x}$. By comparing Figs. 16, 18 and 20(a), it can be concluded that the matched filtering and RDF techniques are saturated with smaller $N_{F}$ s than the FFT-IFFT technique. This means that in the matched filtering and RDF techniques, zeropadding affects the results less. The reason for this can be found in (13) and (19). FTs in (13) and (19) change only the computational domain from spatial to Fourier. This is different for (12). In (12), increasing the number of FFT points (applying zero-padding) affects the discretization of the wavenumbers $k_{x}$ and $k_{y}$. In fact, the greater the number of FFT points, the better the resolution of the wavenumber domain, which is reflected in the reconstructed images. Another point is that in our example, the size of the raw data $(76 \times 64)$ is relatively small compared to the more practical scenarios. Therefore, very large $N_{F}$ values will not be very effective here. Obviously, for scenarios with larger data sizes (more antennas and more scanning points), relatively larger $N_{F} \mathrm{~s}$ will be required. In that case, the efficiency of the proposed RDF technique in terms of computational complexity and, consequently, processing speed will be more apparent. Fig. 21 shows the images reconstructed by the approach [11] for $M_{x}=M_{y}=25$ and $M_{x}=M_{y}=175$. It can be clearly seen that the number of pixels intended for scene discretization greatly affects the quality of the reconstructed images. Significant improvement in Fig. 21(b) compared to Fig. 21(a) comes at the cost of approximately forty times the computational time cost as shown in Fig. 14(b). Improvement in the reconstructed images saturates after reaching an upper- bound limit for $M_{x}$ and $M_{y}$. In our experiments, in this particular case, this saturation behavior was evident when $M_{x}$ and $M_{y}$ dimensions reached approximately $201 \times 201$ (see Fig. 22). The reference image for the diagrams in Fig. 22 is the image reconstructed by approach [11] in first mode (Setup 1, wide beam horn). Although Fig. 21(b) gives almost the best image quality obtained from the approach [11], some distortion is still visible at the target edges. A fairer comparison between the performance of the approach [11] and the proposed approach is the comparison between Figs. 21(a) and 11(a). This comparison is fair because the images of these figures have been reconstructed with the same configuration and almost the same processing time. Even with a cursory glance, one can infer the superiority of the quality of the reconstructed image in Fig. 11(a). Table III presents a comparison between the computational time of image reconstruction by different methods in conditions that have provided almost their best quality. As can be seen, the proposed approach still has a significant advantage in terms of computational time compared to the approach [11]. In addition, by comparing Tables II and III, it can be concluded that by considering larger values for the control parameters (which results in better image quality), the proposed approach (especially by using the RDF technique) further reveals its computational advantage.

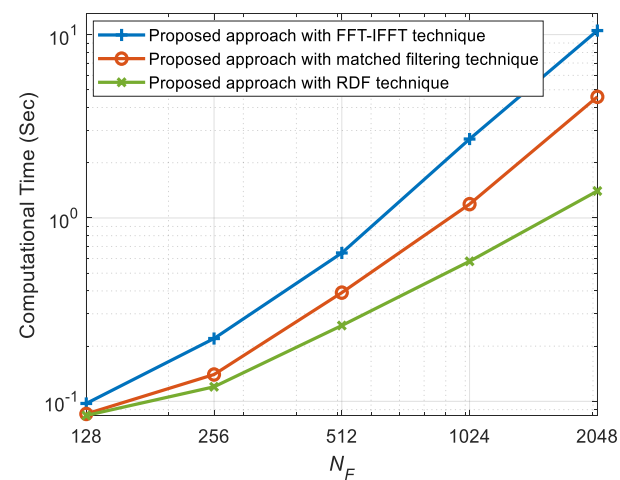

(a)

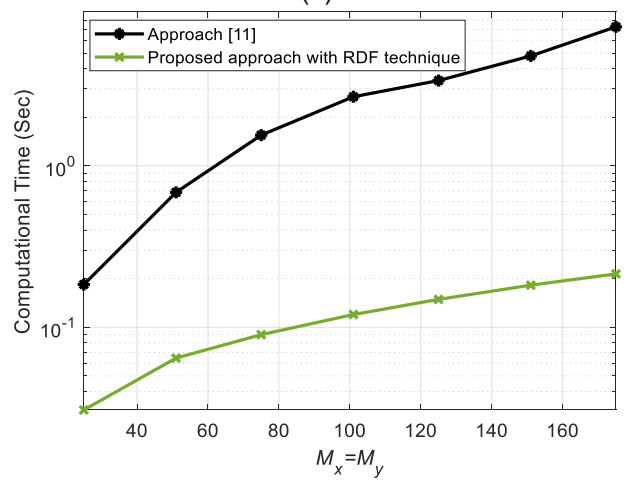

(b)

Fig. 14. Comparison of computational times in different techniques; (a) versus control parameter $N_{F}$ (when $M_{x}=101$ ); (b) versus control parameters $M_{x}$ and $M_{y}$ (when $\left.N_{F}=256\right)$. 
This article has been accepted for publication in a future issue of this journal, but has not been fully edited. Content may change prior to final publication. Citation information: DOI10.1109/TFUZZ.2022.3144448, IEEE Sensors Journal

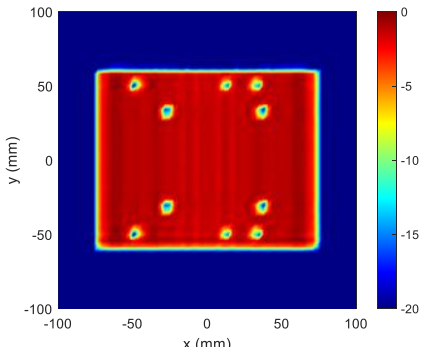

(a)

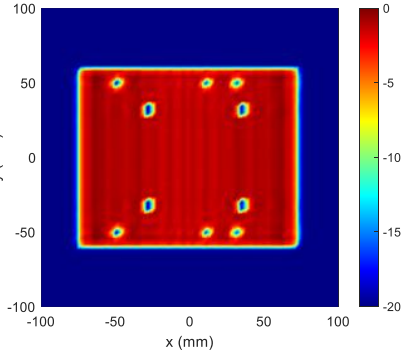

(b)

Fig. 15. Images reconstructed by the proposed approach with FFTIFFT technique using Setup 1 ; (a) $N_{F}=128$, (b) $N_{F}=1024$.

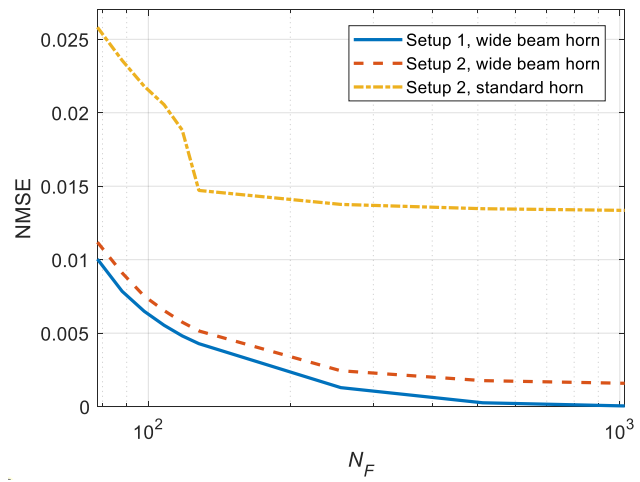

Fig. 16. The NMSE values calculated in the proposed approach with the FFT-IFFT technique in three different modes.

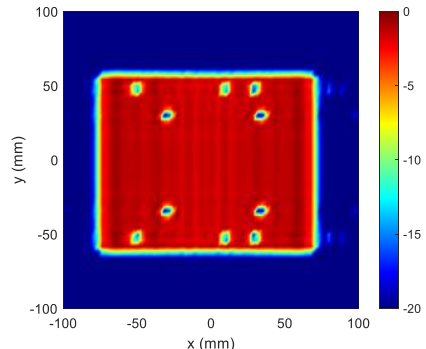

(a)

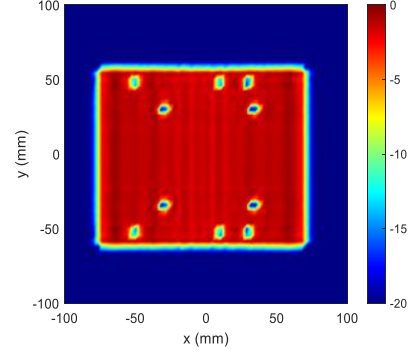

(b)

Fig. 17. Images reconstructed by the proposed approach with matched filtering technique using Setup 1 ; (a) $N_{F}=108$, (b) $N_{F}=512$.

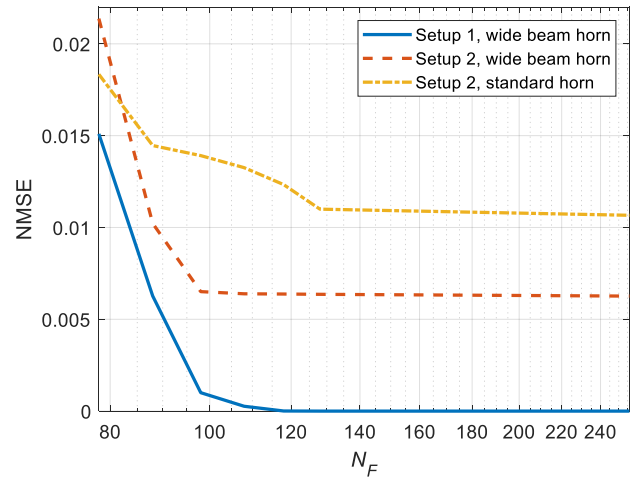

Fig. 18. The NMSE values calculated in the proposed approach with the matched filtering technique in three different modes.

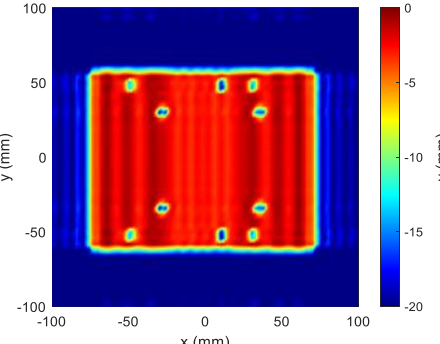

(a)

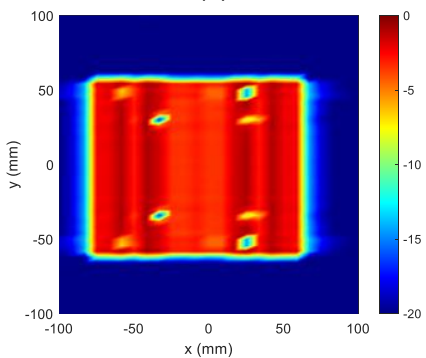

(c)

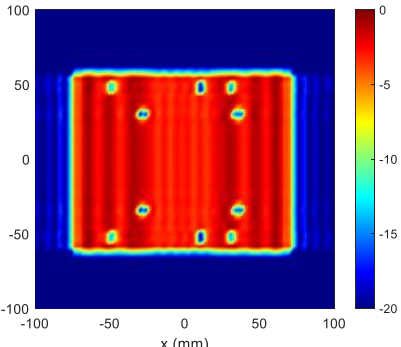

(b)

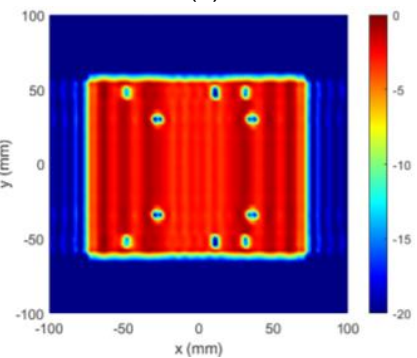

(d)
Fig. 19. Images reconstructed by the proposed approach with RDF technique using Setup 1; (a) $N_{F}=88$ and $M_{x}=101$, (b) $N_{F}=512$ and $M_{x}=101$, (c) $N_{F}=256$ and $M_{x}=25$, (d) $N_{F}=256$ and $M_{x}=175$.

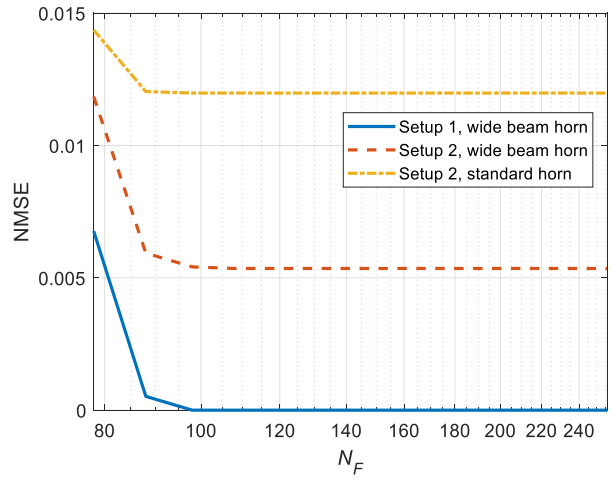

(a)

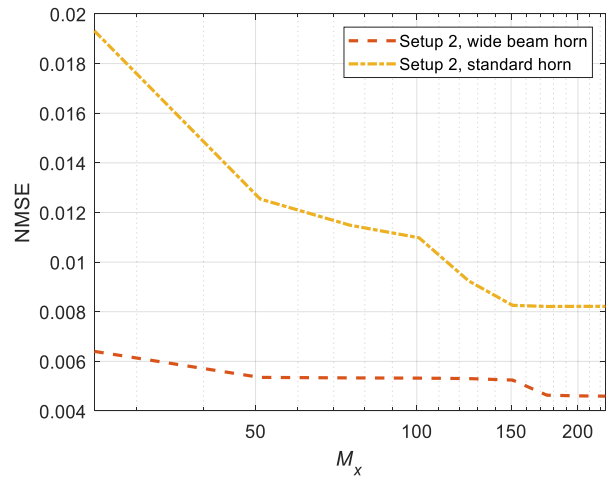

(b)

Fig. 20. The NMSE values calculated in the proposed approach with the RDF technique; (a) versus $N_{F}$ when $M_{x}=101$, (b) versus $M_{x}$ when $N_{F}=256$. 
This article has been accepted for publication in a future issue of this journal, but has not been fully edited. Content may change prior to final publication.

Citation information: DOI10.1109/TFUZZ.2022.3144448, IEEE Sensors Journal

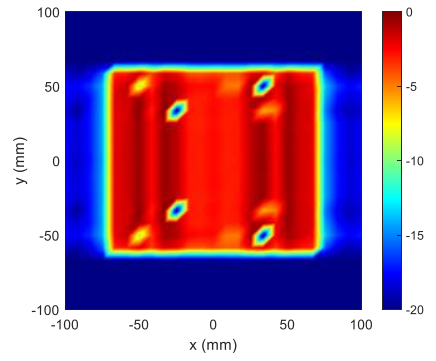

(a)

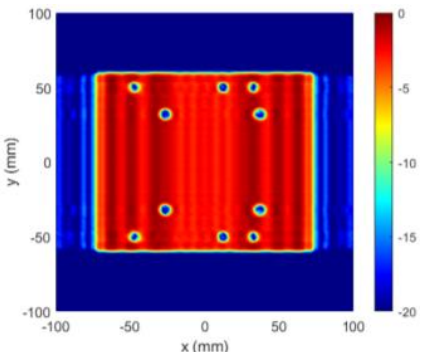

(b)
Fig. 21. Images reconstructed by the approach [11] using Setup 1; (a) $M_{x}=M_{y}=25$, (b) $M_{x}=M_{y}=175$.

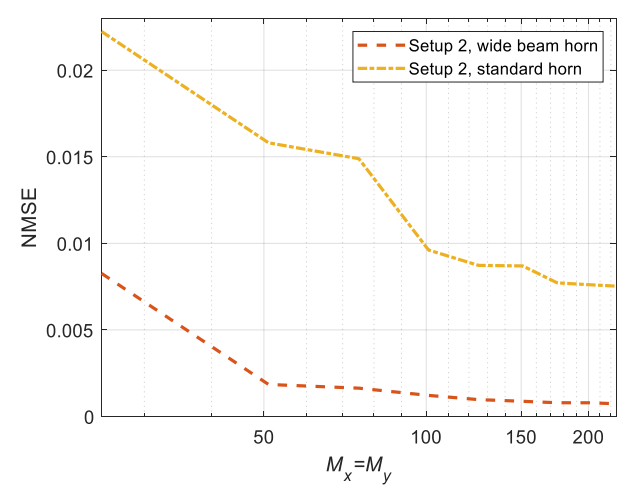

Fig. 22. The NMSE values calculated in the approach [11].

TABLE III

COMPARISON OF COMPUTATIONAL TIMES FOR IMAGE RECONSTRUCTION BY DIFFERENT TECHNIQUES IN FIGS. 15(B), 17(B), 19(D) AND 21(B)

\begin{tabular}{|c|c|c|c|c|}
\hline Figure & $15(\mathrm{~b})$ & $17(\mathrm{~b})$ & $19(\mathrm{~d})$ & $21(\mathrm{~b})$ \\
\hline Computational Time & $2.69 \mathrm{Sec}$ & $0.39 \mathrm{Sec}$ & $0.21 \mathrm{Sec}$ & $7.26 \mathrm{Sec}$ \\
\hline
\end{tabular}

In order to verify the performance, we have also tested the proposed approach with experimental data. Fig. 23 shows photos of the entire system setup and a closer view of the mounted target. In the experimental setup, as $\mathrm{Tx}$ and $\mathrm{Rx}$ antennas, horn antennas are used. The horns used in the experiment are standard pyramidal horns in the $220-325 \mathrm{GHz}$ band with a peak gain of about $23.81 \mathrm{dBi}$ at $220 \mathrm{GHz}$ and a $13 \mathrm{dBm}$ typical output power from the Tx at $220 \mathrm{GHz}$. To perform vertical scanning, the target is mounted on the head of the PNF-XYV-0.9x0.9 system and moves vertically, which inversely imitates a relatively mechanical scanning of the array to a fixed target (similar to an inverse synthetic aperture radar). The system includes a software workstation preloaded with NSI2000 antenna measurement software and controls the stepper motors and experimental parameters (step size, frequency, etc.). In order to realize a linear SPA, two $\mathrm{THz}$ mixer heads are mounted on two linear horizontal scanning stages. To make sure that the placement of the Tx and Rx antennas can be practically feasible, Setup 2 as depicted in Fig. 2(b), is implemented for the experimental study, where the $\mathrm{Tx}$ and $\mathrm{Rx}$ antennas are separated by a $181 \mathrm{~mm}$ gap, moving to each $\mathrm{Tx}$ and $\mathrm{Rx}$ element position in the SPA. Ideally, we should keep this gap as small as possible, and $181 \mathrm{~mm}$ is the minimum gap to place both $\mathrm{Tx}$ and $\mathrm{Rx}$ heads, and allow them to move smoothly within the experimental setup [11]. As we have shown in the results based on numerical data, this vertical gap between the $\mathrm{Tx}$ and $\mathrm{Rx}$ elements has a relatively minor effect on the reconstructed images. This experimental setup uses only one $\mathrm{Tx}$ and $\mathrm{Rx}$ channel to imitate the Tx and Rx arrays. This mechanism not only avoids the use of a large number of real channels with high cost but also eliminates the phase calibration issue in the experiment. In addition, this setup can provide a possible solution for the low-cost practical implementation of the $\mathrm{THz}$ SPA imaging system. Note that the synthesized aperture is a collection of measurements collected at each sampling point across the synthesized aperture. Although each sampling point can be considered a single-input single-output case, the synthesized aperture is a MIMO system. In other words, we are imitating the imaging setup in Fig. 2 by mechanically scanning the Tx and Rx channels and treating each sampling as a separate measurement. This is identical to using an array of antennas and switching between the antennas (similar to the TDM method). Hence, the synthesized aperture is formed by using multiple Tx antennas (output) and multiple Rx antennas (input), i.e. a MIMO aperture. As far as the algorithm is concerned, both these apertures, when considering the overall data, represent an identical MIMO system. Accurately calculating the distance of the imaged object is important particularly at such high frequencies. For experimental measurements, we used a laser distance meter to calculate the distance between the antenna and the imaged object, which is a very accurate way of measuring it. The accuracy of the laser meter is $\pm 0.1 \mathrm{~mm}$, which is less than $\lambda / 10$ at $220 \mathrm{GHz}$. We took the necessary steps to ensure that the characterization of this distance was accurate (our following results will confirm this). An error analysis of the experimental system has been performed in [30] which shows how such errors can affect the reconstruction (particularly Fig. 8 in [30]). It should be noted that this error will only be amplified at $\mathrm{THz}$ frequencies.

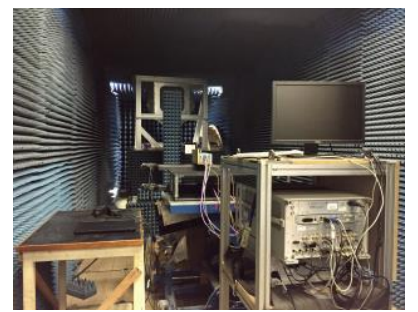

(a)

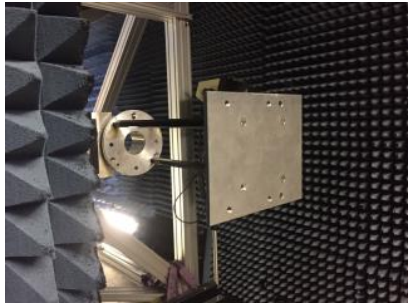

(b)
Fig. 23. Lab setup photos; (a) entire system setup, (b) a closer view of the mounted target.

Fig. 24 shows the reconstructed images using experimental data. As can be seen, the overall structure of the target has been properly reconstructed; however, compared to the simulated numerical data, the image quality deteriorated, so that some holes are not well recognizable. This is due to the narrow beamwidth and the limited sensitivity of the receiver (which in practice leads to a narrower FOV) [31]. In another experiment, we have set the target at $z_{0}=1.4 \mathrm{~m}$ to investigate the effect of the distance from the target to the system on the results. As Fig. 25 shows, increasing the distance has improved the image quality. Although this distance change leads to a tolerable degradation in resolution, by bringing the target closer to the FF region, the accuracy of the approximations used in the multistatic-to-monostatic conversion and interpolation steps is improved (see $[9,15]$ 
and the analyzes in Section III-C).

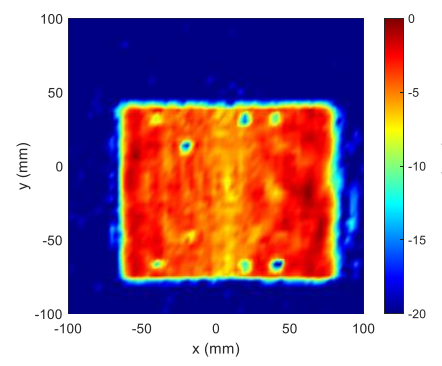

(a)

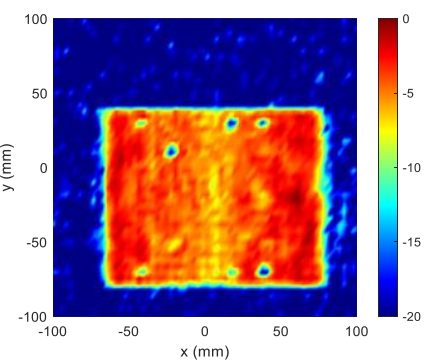

(b)

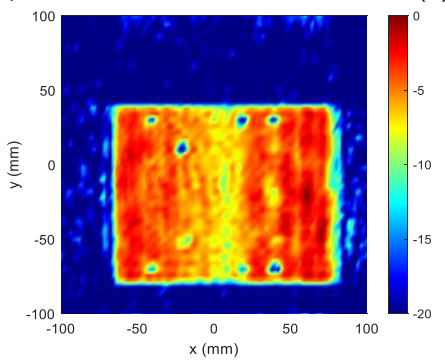

(c)

Fig. 24. Images reconstructed by the proposed approach with standard horn using Setup 2 (based on experimental data); (a) with FFT-IFFT technique, (b) with matched filtering technique, (c) with RDF technique.

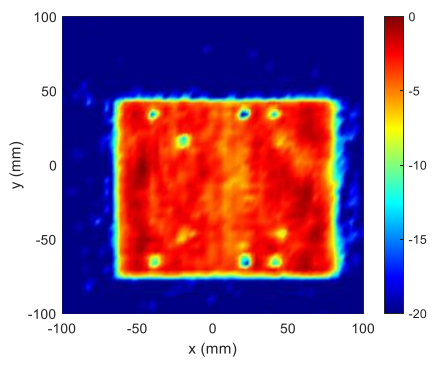

(a)

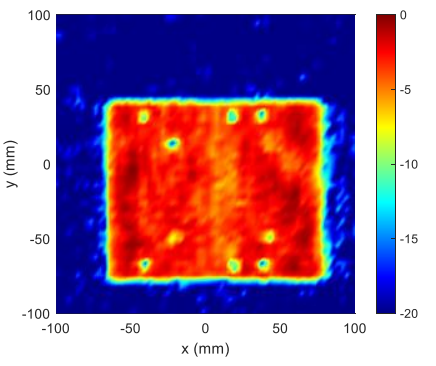

(b)

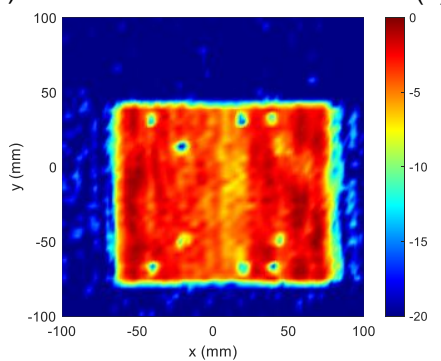

(c)

Fig. 25. Reconstructed images corresponding to Fig. 24, when the target distance from the system has increased by $0.3 \mathrm{~m}$; (a) with FFTIFFT technique, (b) with matched filtering technique, (c) with RDF technique.

The equations derived and employed to reconstruct the images in this paper are not defined on the basis of a particular type of object (see, for example, (12), (13), and (19)). Therefore, considering the appropriate size for the target, the equations derived in this paper can be used for imaging any kind of target. The purpose of applying a fixed target in previous experiments was to make a fair and one-to-one comparison in different scenarios (with Setups 1 and 2, with wide beam horn and standard horn), with different parameters (number of FFT points, number of pixels, spacing between elements, and range) and various techniques (GSAFT [11], matched filtering, FFT-IFFT and RDF). However, here, to further ensure the performance of the proposed approach, we test another target with a different aperture size. To this end, a pure metallic target with a width of $0.8 \mathrm{~m}$ is considered (see Fig. 26). The size of the rectangular holes in the scene from small to large are $3 \times 5,5 \times 7,7 \times 10$ and $10 \times 15 \mathrm{~mm}^{2}$, respectively. The dimensions of large squares and the squares inside them are $30 \times 30$ and $10 \times 10 \mathrm{~mm}^{2}$, respectively. The parameters of the imaging system under the Setup 1 configuration are given in Table IV. Fig. 27 shows the image of the raw echo data obtained from the electromagnetic simulation. Similar to what we did with the first target, here, too, we first examine the effect of the multistatic-tomonostatic conversion and the phase interpolation steps presented in the proposed approach on the results. Fig. 28(a) shows the reconstructed image resulting from applying the FFT-IFFT operation directly to the raw data. As expected, due to the multistatic structure of the imaging and the presence of the target in the NF, the conventional FFT-IFFT method has not been able to create a meaningful image of the scene. However, when we apply the FFT-IFFT operation to the converted data $\tilde{\mathbf{S}}$, the results give a good idea of the target (see Fig. 28(b)). Finally, after another processing step, and using phase interpolation, we were able to further improve the image quality (see Fig. 29(a)). Also, the results of employing matched filtering and RDF techniques in the proposed approach are given in Figs. 29(b) and 29(c), respectively. In addition, Fig. 29(d) shows the image reconstructed by the approach [11]. In all of the images in Fig. 29, the objects in the center show a better resolution than the objects on the side (which are copies of the central objects). The reason for this can be found by comparing the central and lateral parts (along the horizontal axis) of Fig. 27. As can be seen, the points closer to the center of the target area have the highest intensity. In general, moving away from the center of the field usually causes a drop in resolution [32]. Table V presents the computational times calculated for the reconstructed images in Fig. 29. It can be seen from Table $\mathrm{V}$ that the proposed approach is still much more cost-effective than the approach [11] in terms of processing speed. The reason why, unlike previous experiments, the computational time of the RDF technique is slightly longer than that of the matched filtering technique is that in previous experiments the number of physical antennas was lower (8 $\mathrm{Tx}$ and $8 \mathrm{Rx}$ antennas); while in the recent experiment, the number of antennas is almost doubled (14 $\mathrm{Tx}$ and $16 \mathrm{Rx}$ antennas). According to the analyzes performed in Section III-D, the computational complexity of RDF is directly related to the number of antennas, whereas this is not the case for FFT-IFFT and matched filtering techniques. However, RDF still has the advantage of simpler Fourier calculations with less memory required (see Section III-D for more details).

\section{CONCLUSION}

In this paper, a fast processing approach compatible with linear SPA was presented for $\mathrm{NF} \mathrm{THz}$ imaging. We first employed a multistatic-to-monostatic conversion to reduce 
This article has been accepted for publication in a future issue of this journal, but has not been fully edited. Content may change prior to final publication. Citation information: DOI10.1109/TFUZZ.2022.3144448, IEEE Sensors Journal

Molaei et al.: Fast Processing Approach for Near-Field Terahertz Imaging with Linear Sparse Periodic Array

phase errors due to NF multistatic imaging. To improve the quality of the results, we mathematically derived an interpolation formula to counteract the non-uniform spacing of the virtual array. Then, we processed the modified data by three rapid techniques (FFT-IFFT, matched filtering and a new technique with low computational complexity called RDF) to obtain reconstructed images of the scene. We examined and discussed the performance of the proposed approach with numerous data from electromagnetic simulation in FEKO as well as experimental data. The results confirmed the satisfactory performance of the proposed approach in terms of both computational time and the quality of the reconstructed image. In cases where processing speed is a priority, employing the RDF technique in the proposed approach will be more effective; however, in cases where the quality of the reconstructed image has a higher priority, employing the FFTIFFT technique in the proposed approach is recommended. The proposed approach can be effective for real-time imaging applications such as concealed threat detection in high foot traffic environments [33], image-guided surgery [34] and military vetronic systems [35], in which end-users need fast processed data to make decisions.

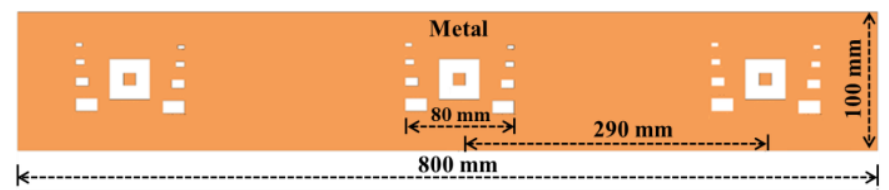

Fig. 26. Second target profile. All the results presented in Figs. 27-29 are related to this target profile.

TABLE IV

IMAGING SYSTEM PARAMETERS FOR THE TARGET IN FIG. 26

\begin{tabular}{|c|c|c|c|c|c|c|c|c|}
\hline Parameter & $f_{0}$ & $N_{t}$ & $N_{r}$ & $N_{y}$ & $d_{t}$ & $d_{r}$ & $d_{y}$ & $z_{0}$ \\
\hline Value & $220 \mathrm{GHz}$ & 14 & 16 & 81 & $7 \mathrm{~mm}(5.13 \lambda)$ & $49 \mathrm{~mm}(35.93 \lambda)$ & $6 \mathrm{~mm}$ & $4 \mathrm{~m}$ \\
\hline
\end{tabular}

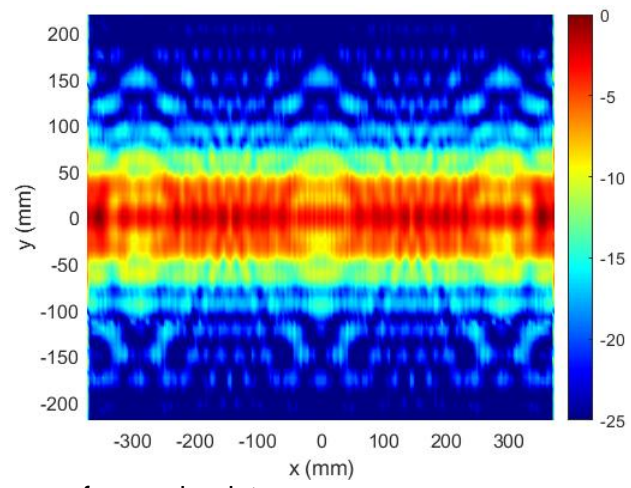

Fig. 27. Image of raw echo data.

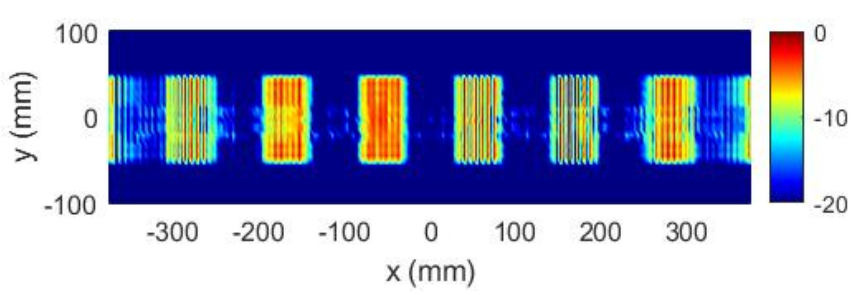

(a)

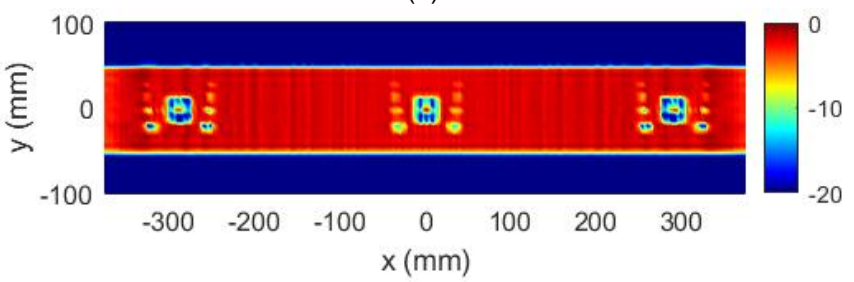

(b)

Fig. 28. Reconstructed images; (a) by applying FFT-IFFT operations directly to $\mathbf{S}$; (b) by applying FFT-IFFT operations to $\tilde{\mathbf{S}}$.

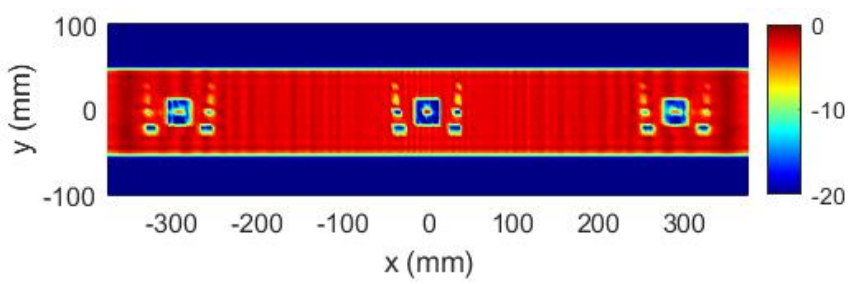

(a)

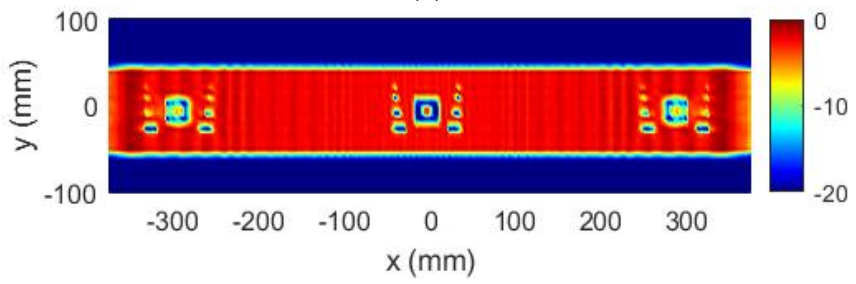

(b)

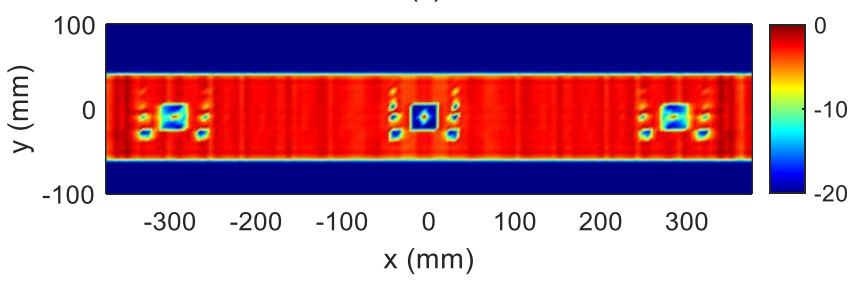

(c)

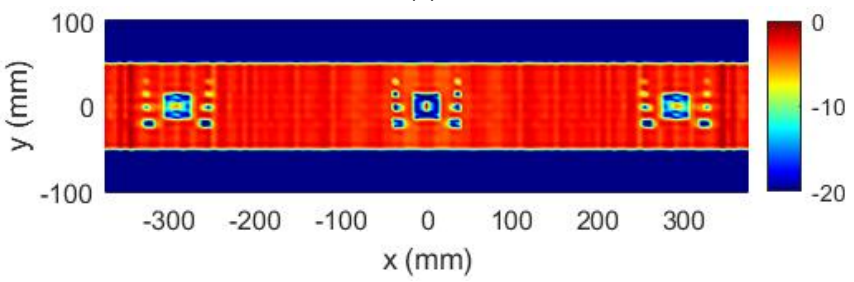

(d)

Fig. 29. Reconstructed images. $N_{F}=256$ and $M_{x}=M_{y}=125$; (a) by the proposed approach with FFT-IFFT technique, (b) by the proposed approach with matched filtering technique, (c) by the proposed approach with RDF technique, (d) by the approach [11].

TABLE V

COMPARISON OF COMPUTATIONAL TIMES FOR IMAGE RECONSTRUCTION BY DIFFERENT TECHNIQUES IN FIG. 29

\begin{tabular}{|c|c|c|c|c|}
\hline Figure & $29(\mathrm{a})$ & $29(\mathrm{~b})$ & $29(\mathrm{c})$ & $29(\mathrm{~d})$ \\
\hline Computational Time & $0.66 \mathrm{Sec}$ & $0.3 \mathrm{Sec}$ & $0.34 \mathrm{Sec}$ & $10.08 \mathrm{Sec}$ \\
\hline
\end{tabular}




\section{REFERENCES}

[1] N. Sellers, Handbook of Terahertz Technology. Scientific e-Resources, 2018.

[2] V. A. Mikhnev, S. N. Vainshtein, and J. T. Kostamovaara, "TimeDomain Terahertz Imaging of Layered Dielectric Structures With Interferometry-Enhanced Sensitivity," IEEE Transactions on Terahertz Science and Technology, vol. 10, no. 5, pp. 531-539, 2020.

[3] A. Bandyopadhyay and A. Sengupta, "A Review of the Concept, Applications and Implementation Issues of Terahertz Spectral Imaging Technique," IETE Technical Review, pp. 1-19, 2021.

[4] G. C. Trichopoulos, H. L. Mosbacker, D. Burdette, and K. Sertel, "A broadband focal plane array camera for real-time $\mathrm{THz}$ imaging applications," IEEE Transactions on Antennas and Propagation, vol. 61, no. 4, pp. 1733-1740, 2013.

[5] M. Zhou, Y. Alfadhl, and X. Chen, "Optimal spatial sampling criterion in a $2 \mathrm{D} \mathrm{THz} \mathrm{holographic} \mathrm{imaging} \mathrm{system,"} \mathrm{IEEE} \mathrm{Access,} \mathrm{vol.} \mathrm{6,} \mathrm{pp.}$ 8173-8177, 2018.

[6] W. Lee and Y. Roh, "Ultrasonic transducers for medical diagnostic imaging," Biomedical engineering letters, vol. 7, no. 2, pp. 91-97, 2017.

[7] J. Gao, Y. Qin, B. Deng, H. Wang, and X. Li, "Novel efficient 3D shortrange imaging algorithms for a scanning 1D-MIMO array," IEEE Transactions on Image Processing, vol. 27, no. 7, pp. 3631-3643, 2018.

[8] C. Lee, W. Choi, J. Kim, and C. Kim, "Three-dimensional clinical handheld photoacoustic/ultrasound scanner," Photoacoustics, vol. 18, p. 100173, 2020.

[9] F. Gumbmann and L.-P. Schmidt, "Millimeter-wave imaging with optimized sparse periodic array for short-range applications," IEEE Transactions on Geoscience and Remote Sensing, vol. 49, no. 10, pp. 3629-3638, 2011.

[10] S. Hu, X. Chen, and Y. Alfadhl, "A THz imaging system using sparse antenna array for security screening," in 2018 43rd International Conference on Infrared, Millimeter, and Terahertz Waves (IRMMW$\mathrm{THz})$, 2018: IEEE, pp. 1-2.

[11] S. Hu, C. Shu, Y. Alfadhl, and X. Chen, "A THz imaging system using linear sparse periodic array," IEEE Sensors Journal, vol. 20, no. 6, pp. 3285-3292, 2019.

[12] S. Hu, "Study on THz Imaging System for Concealed Threats Detection," School of Electronic Engineering and Computer Science Queen Mary, University ..., 2020.

[13] M. E. Yanik, D. Wang, and M. Torlak, "3-D MIMO-SAR imaging using multi-chip cascaded millimeter-wave sensors," in 2019 IEEE Global Conference on Signal and Information Processing (GlobalSIP), 2019: IEEE, pp. 1-5.

[14] Z. Wang, Q. Guo, X. Tian, T. Chang, and H.-L. Cui, "Near-field 3-D millimeter-wave imaging using MIMO RMA with range compensation," IEEE Transactions On Microwave Theory and Techniques, vol. 67, no. 3, pp. 1157-1166, 2018.

[15] A. M. Molaei, O. Yurduseven, and V. Fusco, "An efficient waveform diversity based on variational mode decomposition of coded beatfrequency shifted signals algorithm for multiple-input multiple-output millimetre-wave imaging," IET Radar, Sonar \& Navigation, 2021.

[16] S. A. Bawazeer, S. S. Baakeem, and A. A. Mohamad, "New Approach for Radial Basis Function Based on Partition of Unity of Taylor Series Expansion with Respect to Shape Parameter," Algorithms, vol. 14, no. 1, p. 1, 2021.

[17] M. E. Yanik and M. Torlak, "Near-field MIMO-SAR millimeter-wave imaging with sparsely sampled aperture data," Ieee Access, vol. 7, pp. 31801-31819, 2019.

[18] S. Patole and M. Torlak, "Two dimensional array imaging with beam steered data," IEEE transactions on image processing, vol. 22, no. 12, pp. 5181-5189, 2013.

[19] M. E. Yanik and M. Torlak, "Millimeter-wave near-field imaging with two-dimensional SAR data," Proc. SRC Techcon, no. P093929, 2018.

[20] J. M. Lopez-Sanchez and J. Fortuny-Guasch, "3-D radar imaging using range migration techniques," IEEE Transactions on antennas and propagation, vol. 48, no. 5, pp. 728-737, 2000.

[21] X. Zhuge and A. G. Yarovoy, "Three-dimensional near-field MIMO array imaging using range migration techniques," IEEE Transactions on Image Processing, vol. 21, no. 6, pp. 3026-3033, 2012.

[22] S. Gu, C. Li, X. Gao, Z. Sun, and G. Fang, "Three-dimensional image reconstruction of targets under the illumination of terahertz Gaussian beam-Theory and experiment," IEEE transactions on geoscience and remote sensing, vol. 51, no. 4, pp. 2241-2249, 2012.
[23] J. Wang, P. Aubry, and A. Yarovoy, "3-D short-range imaging with irregular MIMO arrays using NUFFT-based range migration algorithm," IEEE Transactions on Geoscience and Remote Sensing, vol. 58, no. 7, pp. 4730-4742, 2020.

[24] J. Li, X. Wang, and T. Wang, "On the validity of Born approximation," Progress In Electromagnetics Research, vol. 107, pp. 219-237, 2010.

[25] Y. Álvarez, Y. Rodriguez-Vaqueiro, B. Gonzalez-Valdes, F. Las-Heras, and A. García-Pino, "Fourier-based imaging for subsampled multistatic arrays," IEEE Transactions on Antennas and Propagation, vol. 64, no. 6, pp. 2557-2562, 2016.

[26] Z. Li, J. Wang, and Q. H. Liu, "Interpolation-free Stolt mapping for SAR imaging," IEEE Geoscience and Remote Sensing Letters, vol. 11, no. 5, pp. 926-929, 2013.

[27] H.-C. Zeng, J. Chen, W. Liu, and W. Yang, "Modified Omega-k algorithm for high-speed platform highly-squint staggered SAR based on azimuth non-uniform interpolation," Sensors, vol. 15, no. 2, pp. 3750-3765, 2015.

[28] A. M. Molaei, B. Zakeri, and S. M. H. Andargoli, "A one-step algorithm for mixed far-field and near-field sources localization," Digital Signal Processing, vol. 108, p. 102899, 2021.

[29] W. Chunli, L. Xiaowan, L. Cuili, and L. Shuo, "An improved total variation regularized SENSE reconstruction for MRI Images," in 2017 29th Chinese Control And Decision Conference (CCDC), 2017: IEEE, pp. 5005-5009.

[30] O. Yurduseven, T. Fromenteze, and D. R. Smith, "Relaxation of alignment errors and phase calibration in computational frequencydiverse imaging using phase retrieval," IEEE Access, vol. 6, pp. 1488414894, 2018.

[31] H. Tu et al., "A fast 220-GHz real aperture 3-D personnel screening system with a novel-shaped mirror design," IEEE Transactions on Terahertz Science and Technology, vol. 9, no. 3, pp. 253-261, 2019.

[32] N. J. Sofroniew, D. Flickinger, J. King, and K. Svoboda, "A large field of view two-photon mesoscope with subcellular resolution for in vivo imaging," Elife, vol. 5, p. e14472, 2016.

[33] W. F. Moulder et al., "Mobile Testbed for Video-Rate Multistatic Microwave Imaging Array," in 2018 IEEE International Symposium on Antennas and Propagation \& USNC/URSI National Radio Science Meeting, 2018: IEEE, pp. 3-4.

[34] C. Wang et al., "Optical molecular imaging for tumor detection and image-guided surgery," Biomaterials, vol. 157, pp. 62-75, 2018.

[35] J. Phillips, "Choosing the right video interface for military vision systems," in Image Sensing Technologies: Materials, Devices, Systems, and Applications II, 2015, vol. 9481: International Society for Optics and Photonics, p. 948111. 